\title{
Liposomes containing bile salts as novel ocular delivery systems for tacrolimus (FK506): in vitro characterization and improved corneal permeation
}

\author{
This article was published in the following Dove Press journal: \\ International Journal of Nanomedicine \\ 13 May 2013 \\ Number of times this article has been viewed
}

\author{
Yikang Dai' \\ Rui Zhou ${ }^{2}$ \\ Lin Liu' \\ Yi Lu \\ Jianping $\mathrm{Qi}^{2}$ \\ Wei $\mathrm{Wu}^{2}$ \\ 'Department of Ophthalmology, Renji \\ Hospital, School of Medicine, Shanghai \\ Jiaotong University, ${ }^{2}$ Key Laboratory \\ of Smart Drug Delivery, Ministry \\ of Education and PLA, School of \\ Pharmacy, Fudan University, Shanghai, \\ People's Republic of China
}

\begin{abstract}
The objective of this study was to investigate the potential of liposomes containing bile salts as an ophthalmic delivery system for tacrolimus to improve corneal permeability. Liposomes containing bile salts, including sodium taurocholate, sodium deoxycholate, and sodium glycocholate, were produced by the thin-film dispersion method with a particle size of approximately $100 \mathrm{~nm}$ and an entrapment efficiency of more than $90 \%$. Less than $5 \%$ tacrolimus was released from conventional liposomes and from liposomes containing sodium taurocholate, sodium deoxycholate, or sodium glycocholate over 12 hours. The cellular uptake of conventional liposomes was significantly higher than that of liposomes containing bile salts. However, liposomes containing bile salts exerted a 3-4-fold increase of tacrolimus in ex vivo corneal transport of tacrolimus compared with conventional liposomes. When rabbit eyes were treated with a DiI perchlorate-loaded liposome suspension, liposomes containing bile salts showed fast and sustained penetration across the cornea. Unfortunately, liposomes containing sodium deoxycholate caused toxicity or irritation to both spontaneously derived human corneal epithelial cells and the rabbit cornea. Therefore, liposomes containing sodium taurocholate and sodium glycocholate are potential carriers in ocular drug delivery systems, given their low toxicity and vastly improved permeability.

Keywords: liposomes, bile salt, tacrolimus, cornea, sodium taurocholate, sodium deoxycholate, sodium glycocholate
\end{abstract}

\section{Introduction}

Corneal transplantation has the highest success rate of all types of human organ and tissue transplantations, although immunological rejection remains a major factor in graft failure. ${ }^{1}$ Drug therapy with immunosuppressants, such as cyclosporin $\mathrm{A}^{2}$ and tacrolimus (FK506) ${ }^{3}$, is the main strategy used to inhibit immunological rejection. FK506 is a new immunosuppressant agent isolated by fermentation from Streptomyces tsukubaensis and is similar to cyclosporin A, but is more potent (up to 100 times the potency of cyclosporin A). ${ }^{4}$ Clinical studies have shown that FK506 delivered topically is able to inhibit immunological rejection after high-risk corneal and limbal grafts. ${ }^{5}$ However, FK506 has difficulty penetrating the corneal epithelium and accumulates in the corneal stroma due to its poor water solubility $(1.3 \mu \mathrm{g} / \mathrm{mL})^{6}$ and relatively high molecular weight $(804.02 \mathrm{~g} / \mathrm{mol})$. For inhibition of immunological rejection after corneal grafts, eye drops are a better choice over intraocular dosage formulations. Eye drops in the form of a tacrolimus suspension ${ }^{7}$ and oil solution ${ }^{8}$ have been reported.
Correspondence: Jianping Qi

Department of Pharmaceutics,

School of Pharmacy, Fudan University,

826 Zhangheng Road, Shanghai 20I203,

People's Republic of China

Tel +862151980084

$\mathrm{Fax}+862151980084$

Email qijianping@fudan.edu.cn 
Although eye drops have distinct advantages, such as ease of administration and good compliance, physiological barriers at the cornea lead to poor absorption of drug molecules and consequently poor therapeutic efficacy when used topically. These constraints include poor corneal permeation, elimination by tears and the nasolacrimal duct, and transportation into the systemic circulation by other absorption pathways. ${ }^{9}$ Meanwhile, both suspensions and oil solutions show poor biocompatibility and are strong irritants. Therefore, improving corneal penetration and prolonging precorneal retention time are critical aspects to be considered when designing a formulation. Nanoscale drug delivery systems, such as nanoparticles, ${ }^{10}$ cubosomes, ${ }^{9,11}$ nanoemulsions, ${ }^{12}$ and liposomes, ${ }^{13}$ have been shown to improve corneal penetration and increase retention time. Because of their resemblance to biomembranes, liposomes have improved biocompatibility. A previous study has indicated that liposomes loaded with FK506 can facilitate penetration of the drug across the cornea into the aqueous humor and that the drug would disperse throughout the ocular tissue, similar to an olive oil solution of FK506. ${ }^{8}$ However, the fraction of liposomal FK506 that is absorbed is still too small to achieve therapeutic levels, so the transcorneal permeation of FK506 requires improvement.

Replacing a fraction of the cholesterol in the liposomes with bile salts causes the liposomes to become more flexible, which may facilitate penetration of the vehicle across biomembranes. ${ }^{14}$ Recent reports indicate that liposomes containing bile salts improve the oral ${ }^{15}$ and transdermal absorption ${ }^{16}$ of drug molecules when compared with conventional liposomes. Bile salts used in drug delivery systems include primarily sodium glycocholate, sodium deoxycholate, and sodium taurocholate, because they are less irritating. ${ }^{17}$

In this study, tacrolimus-loaded liposomes containing three types of bile salts (sodium glycocholate, sodium deoxycholate, or sodium taurocholate) were prepared and characterized in vitro. Transcorneal permeation was evaluated in an ex vivo diffusion cell model using excised corneas. Further, we used spontaneously derived human corneal epithelial cells (SDHCECs) to evaluate the uptake and toxicity of liposomes containing bile salts, to assess their potential for ocular application. Finally, in vivo uptake and toxicity were assessed after administration of liposomes to rabbit eyes.

\section{Materials and methods Materials}

Tacrolimus was purchased from Shanghai Baibo Biotechnology Company (Shanghai, People's Republic of China). Sodium glycocholate was purchased from Amresco
(Solon, OH, USA). Soybean phosphatidylcholine (S100) and cholesterol were supplied by Lipoid (Lipoid GmbH, Ludwigshafen, Germany). Sodium taurocholate and sodium deoxycholate were obtained from Sigma (St Louis, MO, USA). Sephadex G-25 was purchased from Pharmacia (Peapack, NJ, USA). DiI perchlorate (1,1'-dioctadecyl-3,3',3'tertamethylin-docarbocyanine perchlorate) was obtained from Fanbo Biochemicals (Shanghai, People's Republic of China). High-performance liquid chromatography grade acetonitrile and methanol were purchased from Tedia Company Inc (Fairfield, CA, USA). Methylthiazolyldiphenyl tetrazolium bromide (MTT) was supplied by Sigma. All other reagents were analytical grade.

\section{Preparation of tacrolimus-loaded liposomes containing bile salts}

Tacrolimus-loaded liposomes containing bile salts were prepared using a thin-film dispersion method. ${ }^{15}$ Briefly, soybean phosphatidylcholine, bile salts (sodium glycocholate, sodium taurocholate, and sodium deoxycholate) and tacrolimus were dissolved in dichloromethane/methanol $(9: 1, v / v)$ in a round-bottom flask. The organic solvent was then removed using a rotary evaporator (RV 10 digital, IKA Works, Staufen, Germany) in a $37^{\circ} \mathrm{C}$ water bath under vacuum. The dried lipid film was maintained under reduced pressure for two hours to remove traces of solvent. The lipid film was then hydrated with $10 \mathrm{~mL}$ phosphate buffer (pH 7.4) for 30 minutes at $37^{\circ} \mathrm{C}$ to obtain a crude dispersion of liposomes. The particle size of the liposomes was further decreased using a probe ultrasonicator (Scientz-IID, Ningbo Scientz Biotechnology Co, Zhejiang, People's Republic of China). The liposome dispersion was stored at $4^{\circ} \mathrm{C}$ until use. Similar procedures were followed using cholesterol in place of bile salts for preparation of conventional liposomes.

\section{Characterization of liposomes}

\section{Particle size and zeta potential}

The particle size and zeta potential of the tacrolimus-loaded liposomes was determined by dynamic light scattering using a Nano ZS zeta sizer (Malvern, Worcestershire, UK) at $25^{\circ} \mathrm{C}$. Three measurements were taken, and the number of runs in each measurement was automatically determined by the software; the results were expressed as the mean \pm standard deviation.

\section{Transmission electron microscopy}

The morphology of the liposomes was observed using transmission electron microscopy (JEM-1230, JEOL, Tokyo, Japan). A drop of the liposome suspension was 
transferred to a 300-mesh carbon-coated copper grid, and the extra water was removed by blotting. A drop of $2 \%$ uranyl acetate was added for 60 seconds to stain the liposomes, and the suspension was dried under ambient conditions. The samples were inspected at an acceleration voltage of $120 \mathrm{kV}$.

\section{Entrapment efficiency}

The entrapment efficiency of tacrolimus in the liposomes was determined using a gel permeation chromatography method. ${ }^{15}$ Briefly, a column $(20 \mathrm{~cm} \times 1 \mathrm{~cm})$ filled with Sephadex G-25 gel was eluted with $200 \mu \mathrm{L}$ of blank liposomes to saturate the column and improve column recovery. Subsequently, tacrolimus-loaded liposomes were mounted and eluted with water at a flow rate of $1 \mathrm{~mL}$ per minute to separate the free tacrolimus from the liposomes. The elution was monitored by measurement of turbidity at a wavelength of $500 \mathrm{~nm}$ using a spectrophotometer (UV-2401, Shimadzu, Tokyo, Japan). ${ }^{15}$ The eluent-containing liposomes were dissolved in methanol and analyzed for tacrolimus by ultraviolet high-performance liquid chromatography. The entrapment efficiency (EE\%) was calculated as: $\mathrm{EE} \%=\mathrm{W}_{\text {liposome }} / \mathrm{W}_{\text {total }}$, where $\mathrm{W}_{\text {lipsome }}$ and $\mathrm{W}_{\text {total }}$ denote the weight of tacrolimus in liposomes and total tacrolimus weight in the liposome dispersion.

\section{In vitro release}

The in vitro release profiles for the tacrolimus-loaded liposomes were investigated using dynamic dialysis. The release medium was freshly prepared, simulating tear fluid with a composition of $\mathrm{NaCl}(0.67 \mathrm{~g}), \mathrm{NaHCO}_{3}(0.20 \mathrm{~g})$, and $\mathrm{CaCl}_{2} \cdot 2 \mathrm{H}_{2} \mathrm{O}(0.008 \mathrm{~g})$ dissolved in $100 \mathrm{~mL}$ of deionized water. ${ }^{18}$ The release medium contained $0.1 \%$ sodium dodecyl sulfate to achieve sink conditions. Initially, $1 \mathrm{~mL}$ of liposomes (tacrolimus $3 \mathrm{mg} / \mathrm{mL}$ ) were transferred into a dialysis tube (Float-A-Lyzer ${ }^{\circledR}$ G2, Spectrum Laboratories, Torrance, CA, USA) with a $100 \mathrm{kDa}$ membrane molecular weight cutoff, which was then placed into $100 \mathrm{~mL}$ of release medium stirred at $100 \mathrm{rpm}$ at $34^{\circ} \mathrm{C} \pm 0.5^{\circ} \mathrm{C}$. At predefined time intervals, $1 \mathrm{~mL}$ of release medium was withdrawn and an equal volume of fresh release medium was added to maintain a constant volume. The tacrolimus content was determined by ultraviolet high-performance liquid chromatography after centrifugation $(10,000 \times \mathrm{g}$ for five minutes).

\section{Cytotoxicity test using SDHCECs Cell cultivation}

SDHCECs from 40 to 60 passages were grown as a monolayer at $37^{\circ} \mathrm{C}$ in a $5 \% \mathrm{CO}_{2} / 95 \%$ air atmosphere, and cells showing more than $97 \%$ viability were used for the experiments. The culture used was Dulbecco modified Eagle's medium, supplemented with $10 \%$ heat inactivated fetal bovine serum, $10 \mathrm{ng} / \mathrm{mL}$ human epidermal growth factor, $5 \mu \mathrm{g} / \mathrm{mL}$ insulin-human transferrin-Se, $0.4 \mu \mathrm{g} / \mathrm{mL}$ hydrocortisone, $2 \mathrm{mM}$ L-glutamine, $100 \mathrm{U} / \mathrm{mL}$ penicillin, and $100 \mu \mathrm{g} / \mathrm{mL}$ streptomycin (all from Gibco, Grand Island, NY, USA). ${ }^{19}$

\section{Cytotoxicity}

Viability of the SDHCECs was measured by MTT to evaluate the cytotoxicity of liposomes containing bile salts. Approximately $1 \times 10^{4}$ cells $(200 \mu \mathrm{L}$ of cell culture medium, $5 \times 10^{4}$ cells $/ \mathrm{mL}$ ) were seeded in a 96-well tissue culture plate. The cells were then cultured under the same conditions as described for cell cultivation over 24 hours. The medium was removed prior to the experiment and $200 \mu \mathrm{L}$ of liposomes in supplement-free culture medium were added to each well as a negative control. After 10 hours, $20 \mu \mathrm{L}$ of $5 \mathrm{mg} / \mathrm{mL}$ MTT solution was added to each well, and the medium was removed after two hours. The resulting formazan crystals were then dissolved in $150 \mu \mathrm{L}$ of dimethyl sulfoxide, and absorbance was measured at $570 \mathrm{~nm}$.

\section{Uptake by cells}

SDHCECs were seeded in a 24 -well plate at a density of $3 \times 10^{4}$ cells/well and incubated for 24 hours to allow them to adhere. To quantify uptake of the tacrolimus-loaded liposomes by cells, the SDHCECs were incubated with tacrolimus-loaded liposomes in supplement-free culture medium for different time periods (2, 4, 8 and 12 hours). After washing the cells three times with phosphate-buffered saline (pH 7.4), $200 \mu \mathrm{L}$ of phosphate-buffered saline was added, and the cell suspension was freeze-thawed. The freeze-thawed cell suspension $(100 \mu \mathrm{L})$ was vortexed with $100 \mu \mathrm{L}$ of methanol for five minutes, and centrifuged at $10,000 \times \mathrm{g}$ for 10 minutes. The drug concentration in the supernatant was measured by high-performance liquid chromatography tandem mass spectrometry, and the protein content in the cell suspensions was measured using a bicinchoninic acid assay kit (Xitang Biotechnology Company, Shanghai, People's Republic of China). The cellular uptake of tacrolimus was calculated as: drug uptake $(\mathrm{ng} / \mu \mathrm{g}$ protein $)=\mathrm{C}_{\mathrm{TLM}} / \mathrm{C}_{\text {protein }}$, where $\mathrm{C}_{\mathrm{TLM}}$ is the intracellular concentration of tacrolimus and $\mathrm{C}_{\text {protein }}$ is the concentration of cellular protein.

\section{Ex vivo transport across rabbit cornea}

Ex vivo corneal penetration studies were carried out using a Valia-Chien diffusion cell with a diffusion area of $0.825 \mathrm{~cm}^{2}$. Albino New Zealand rabbits weighing 2.0-2.5 kg were obtained from the Laboratory Animal Service Center of Fudan University. All animal treatments were in accordance with 
the regulations for the administration of affairs concerning experimental animals and were approved by the animal ethics committee of Fudan University. The rabbits were euthanized by injection of an overdose of air into the marginal ear vein after being anesthetized with an intramuscular overdose of an anesthetic mixture of xylazine $20 \mathrm{mg} / \mathrm{kg}$ and ketamine $200 \mathrm{mg} / \mathrm{kg}$. The corneas were excised from the globes and mounted onto the ring of the perfusion apparatus. The corneas were gently rinsed with saline, and extreme care was taken not to produce wrinkles or folding in the membrane before mounting. Simulated tear fluid $(3 \mathrm{~mL})$, prewarmed to a temperature of $34^{\circ} \mathrm{C}$, was placed into the receptor chamber of the apparatus, with magnetic stirring throughout the entire experiment. Three milliliters of liposomes (containing $1 \mathrm{mg} / \mathrm{mL}$ tacrolimus) were placed in the donor chamber, which was then sealed to avoid evaporation. A mixture of $\mathrm{O}_{2}: \mathrm{CO}_{2}$ (95:5) was bubbled through the chambers. The hydration level of the cornea, which indicated the corneal condition, was measured according to a method reported previously. ${ }^{20}$

Next, $500 \mu \mathrm{L}$ samples were taken at 30, 60, 90, 120, 180 and 240 minutes and replaced with fresh simulated tear fluid. The amount of drug permeating across the cornea was assayed by high-performance liquid chromatography tandem mass spectrometry.

The amount of drug that permeated through the corneal epithelium was plotted versus time for each liposome and the slope of the linear portion of the graph was calculated. The apparent corneal permeability coefficient $(\mathrm{cm} / \mathrm{s})$ was determined according to the equation: $P_{a p p}=\Delta Q / \Delta \mathrm{t} \cdot \mathrm{C}_{0} \cdot \mathrm{A} \cdot 60$, where $\Delta Q / \Delta \mathrm{t}$ is the linear portion of the slope ( $\mu \mathrm{g}$ per minute), 60 is the conversion constant from minute to seconds, $\mathrm{A}$ is the corneal surface area, and $\mathrm{C}_{0}$ is the initial drug concentration $\left(\mu \mathrm{g} / \mathrm{cm}^{3}\right)$.

\section{In vivo corneal uptake}

In vivo corneal uptake of liposomes was evaluated using fluorescence-labeled frozen sections. The liposome formulation was labeled by adding a specified amount of DiI perchlorate and then processed via the same procedures as those used for preparation of the liposomes containing tacrolimus. The rabbits were randomly divided into four groups of three animals each, and each received $40 \mu \mathrm{L}$ of liposomes containing DiI perchlorate $50 \mu \mathrm{g} / \mathrm{mL}$ in both eyes. At 10, 30, and 60 minutes after instillation, one rabbit from each group was euthanized, and the corneas were excised from the globes and gently rinsed with saline. One rabbit was left untreated as the negative control. The corneas were fixed by immersion into Davidson's solution for four hours and then in $30 \%$ sucrose to dehydrate overnight. The corneas were next embedded in optimum cutting temperature compound (Tissue-Tek ${ }^{\circledR}$, Sakura, Torrance, CA, USA), frozen at $-22^{\circ} \mathrm{C}$, sectioned into $5 \mu \mathrm{m}$ slices, and observed under a confocal scanning laser microscope (LSM 510 Meta, Zeiss, Oberkochen, Germany).

\section{In vivo corneal tolerance test}

In vivo corneal tolerance was evaluated in the paraffin sections following hematoxylin and eosin staining for pathology. The rabbits were randomly divided into five groups of three animals each, and each rabbit received $40 \mu \mathrm{L}$ of liposomes containing tacrolimus $1 \mathrm{mg} / \mathrm{mL}$ in the right eye at 30-minute intervals for six hours. The contralateral eye was used as the control and received no treatment. The sixteenth animal was untreated and served as the sham control. Six hours after the first instillation, the rabbits were euthanized, and the corneas were excised from the globes and gently rinsed with saline. The corneas were immersed into Davidson's solution for one hour and fixed with 10\% neutral formalin. Subsequently, they were dehydrated in an alcohol gradient, placed in melted paraffin, and solidified into block form. The cross-sections were cut, stained with hematoxylin and eosin, and observed microscopically for any pathological modifications. ${ }^{21}$

\section{Determination of tacrolimus}

Tacrolimus was determined using an LC-10 ATvp reversedphase ultraviolet high-pressure liquid chromatography (Shimadzu, Kyoto, Japan) method as previously reported. ${ }^{22}$ The system was composed of a binary pump, a tunable ultraviolet detector, a column heater, and a manual injector. The mobile phase was a mixture of acetonitrile/ $0.1 \%$ phosphoric acid solution $(65 / 35, \mathrm{v} / \mathrm{v})$ pumped at a flow rate of $1.0 \mathrm{~mL}$ per minute. The tacrolimus was separated on a C18 column (Eclipse XDB, $4.6 \times 50 \mathrm{~mm}, 5 \mu \mathrm{m}$, Agilent Technologies Inc, Santa Clara, CA, USA), guarded with a refillable precolumn $(\mathrm{C} 18,2.0 \mathrm{~mm} \times 20 \mathrm{~mm}$, Alltech, Nicholasville, KY, USA). The temperature of the column was maintained at $60^{\circ} \mathrm{C}$. The detection wavelength was $220 \mathrm{~nm}$.

High-performance liquid chromatography tandem mass spectrometry was used to measure tacrolimus samples for the cellular uptake and in vitro corneal transport study. ${ }^{23}$ Chromatographic separation was performed on an Agilent 1200 system composing of a quaternary pump, a degasser, an autosampler, and a column heater (Agilent Technologies Inc). The high-performance liquid chromatography column was a Zorbax XDB-C18 $(2.1 \times 50 \mathrm{~mm}, 5 \mu \mathrm{m})$ column from Agilent. The mobile phase comprised $30 \%$ of a water phase 
( $0.1 \%$ formic acid) and $70 \%$ of an organic phase (methanol with $0.1 \%$ formic acid). The flow rate was set at $0.5 \mathrm{~mL}$ per minute. The column was kept at $50^{\circ} \mathrm{C}$ in a column oven. Mass spectrometric detection was performed on a 4000 Q-TRAP liquid chromatography column coupled with a tandem mass spectrometry system using an ESI ion source (Turbo Ionspray) from AB Sciex Company (Framingham, MA, USA) and operated in a negative ionization mode. The ion spray voltage and source temperature were set at -4500 $\mathrm{V}$ and $450^{\circ} \mathrm{C}$, respectively. Ion transitions were monitored from $\mathrm{m} / \mathrm{z} 802.5 \rightarrow 560.5$ for tacrolimus and $790.5 \rightarrow 548.3$ for ascomycin (internal standard).

\section{Statistical analysis}

One-way analysis of variance was performed on all experimental data, and means were compared using the Student's $t$-test at the $5 \%$ level with Statistical Package for Social Sciences version 13.0 software (SPSS Inc, Chicago, IL, USA).

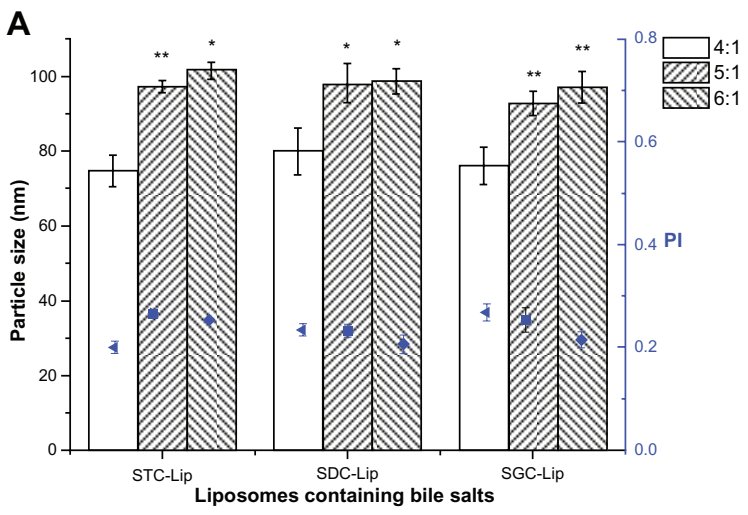

\section{Results and discussion Preparation of tacrolimus-loaded liposomes containing bile salts}

Liposomes containing bile salts were successfully prepared by a thin-film dispersion/sonication method as described in our previous report of encapsulation of cyclosporin A into liposomes containing sodium deoxycholate. ${ }^{15}$ Our present study results indicate that liposomes containing the three different bile salts were not significantly different in particle size or entrapment efficiency $(P>0.05$, Figure $1 \mathrm{~A}$ and $\mathrm{B})$. However, the ratio of soybean phosphatidylcholine to bile salts could affect the particle size and entrapment efficiency of liposomes. The particle size of the liposomes containing 4/1 of soybean phosphatidylcholine to bile salts was significantly lower than that of the other two groups, and liposomes containing $5 / 1$ of soybean phosphatidylcholine to bile salts showed the highest entrapment efficiency. The same trend was observed for each of the three types of

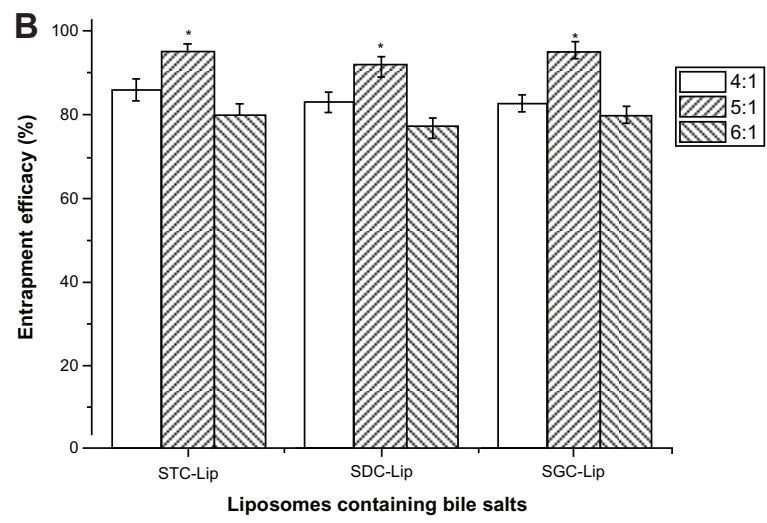

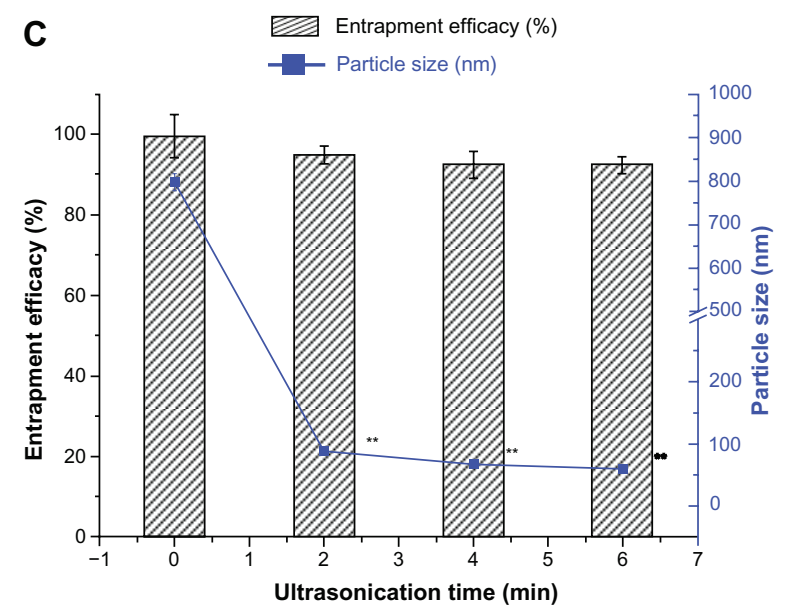

Figure I Effect of formulation (A and B) and ultrasonication time (C) on particle size, polydispersity index (PI), and entrapment efficiency of liposomes (Lip) containing sodium taurocholate (STC), sodium deoxycholate (SDC), or sodium glycocholate (SGC) $(n=3)$.

Notes: $* P<0.05$ and $* * P<0.01$ represent significant differences in particle size and entrapment efficiency between the different SPC/BS ratios in comparison with the particle size of SPC/BS at a ratio of 4/I. **P $<0.01$ represents a significant difference in particle size between different ultrasonication times when compared with no ultrasonication.

Abbreviations: SPC, soybean phosphatidylcholine; BS, bile salts. 
liposomes (ie, those containing sodium taurocholate, sodium deoxycholate, or sodium glycocholate). With an increase in bile salt content, the surface stretching of liposomal systems should decrease and lead to reduced particle size. However, an extremely high content of bile salts increased the solubility of tacrolimus in the dispersion phase, which compromised the entrapment efficiency. The low entrapment efficiency seen for liposomes containing 6/1 soybean phosphatidylcholine to bile salts was possibly the result of poor stability of the liposomes at this ratio. Therefore, we selected $5 / 1$ of soybean phosphatidylcholine to bile salts to prepare the liposomes (containing sodium taurocholate, sodium deoxycholate, or sodium glycocholate bile salts) for further evaluation.

Ultrasonication is frequently used to decrease the size of nanoparticles. Figure $1 \mathrm{C}$ shows the effect of ultrasonication time on particle size and entrapment efficiency. There was a significant reduction in size from approximately $800 \mathrm{~nm}$ to $90 \mathrm{~nm}$ following ultrasonication for two minutes, at an energy input of $380 \mathrm{~W}$. For the duration investigated, time did not seem to affect the particle size. However, ultrasonication showed little effect on the entrapment efficiency for tacrolimus in liposomes, which could be attributed to the lipophilicity of tacrolimus and its high affinity for lipid bilayers.

Table 1 shows the particle size, zeta potential, and entrapment efficiency of tacrolimus liposomes prepared under optimized conditions. Because particle size exerts a significant influence on the performance of liposomes both in vitro and in vivo, it is necessary to ensure consistency of particle size among all the batches of liposomes. With regard to the conventional liposomes, less cholesterol was incorporated to produce liposomes of a smaller particle size because increased cholesterol in the lipid bilayers improves the rigidity of liposomes, which consequently increases the overall particle size of the vesicles. Of note, the negatively charged bile salts stimulated the zeta potential of the liposomes containing the three types of bile salts when compared with liposomes containing cholesterol.

\section{Morphology}

Figure 2 shows transmission electron micrographs for the liposomes. All liposomes were close to spherical in shape, with an average particle size of approximately $100 \mathrm{~nm}$, which correlates well with the results obtained by dynamic light scattering. The vesicles of liposomes containing cholesterol had a tendency to agglomerate, but not those containing bile salts. This phenomenon could be readily attributed to the high zeta potential value, which hindered aggregation of particles. ${ }^{24}$

\section{In vitro release}

Figure 3 shows the in vitro release profiles for the tacrolimus liposomal formulations. The overall release of tacrolimus from the four types of tacrolimus-loaded liposomes was very slow, at no more than $5 \%$ by 24 hours, which was similar to our previous findings with cyclosporine A-loaded liposomes containing sodium deoxycholate. ${ }^{15}$ Because the solubility of tacrolimus in $0.1 \%$ sodium dodecyl sulfate solution was determined to be more than $0.5 \mathrm{mg} / \mathrm{mL}$, good sink conditions could be maintained throughout the release test. Tacrolimus in solution could completely diffuse across the dialysis tube within one hour, indicating a negligible effect of the dialysis tube on release of tacrolimus. The slow release of tacrolimus from the liposomes could be attributed to the high affinity of tacrolimus for the hydrophobic materials in the formulations.

\section{Cytotoxicity}

Viability of SDHCECs after exposure to tacrolimus-loaded liposomes at different concentrations was evaluated directly by MTT assay (Figure 4). Cell viability was more than $85 \%$ for all four types of liposome formulations after 12 hours of incubation, with a phospholipid concentration of less than $4.0 \mathrm{mg} / \mathrm{mL}$. No significant difference in cell viability

Table I Formulation details for tacrolimus-loaded conventional liposomes and liposomes containing sodium taurocholate, sodium deoxycholate, or sodium glycoholate for in vitro and in vivo studies $(n=3)$

\begin{tabular}{lllcccc}
\hline $\begin{array}{l}\text { Liposome } \\
\text { formulation }\end{array}$ & $\begin{array}{l}\text { SPC/BS (CH) } \\
\text { ratio }\end{array}$ & TLM loading (\%) & Particle size (nm) & Polydispersity & Zeta potential (mV) & $\begin{array}{l}\text { Entrapment } \\
\text { efficiency (\%) }\end{array}$ \\
\hline STC-Lip & $5 / I$ & 5.9 & $97.3 \pm 1.6$ & $0.267 \pm 0.033$ & $-25.2 \pm 3.8$ & $95.15 \pm 1.91$ \\
SDC-Lip & $5 / 1$ & 5.9 & $98.1 \pm 5.2$ & $0.233 \pm 0.013$ & $-27.8 \pm 4.9$ & $92.11 \pm 1.66$ \\
SGC-Lip & $5 / I$ & 5.9 & $92.8 \pm 3.2$ & $0.254 \pm 0.023$ & $-21.9 \pm 3.1$ & $94.97 \pm 2.25$ \\
CH-Lip & I2/I & 5.9 & $108.3 \pm 2.6$ & $0.205 \pm 0.017$ & $-1.2 \pm 0.3$ & $96.53 \pm 3.18$ \\
\hline
\end{tabular}

Abbreviations: BS, bile salts; CH-Lip, liposomes containing cholesterol; SGC-Lip, liposomes containing sodium glycocholate; SDC-Lip, liposomes containing sodium deoxycholate; STC-Lip, liposomes containing sodium taurocholate; SPC, soybean phosphatidylcholine; TLM, tacrolimus. 

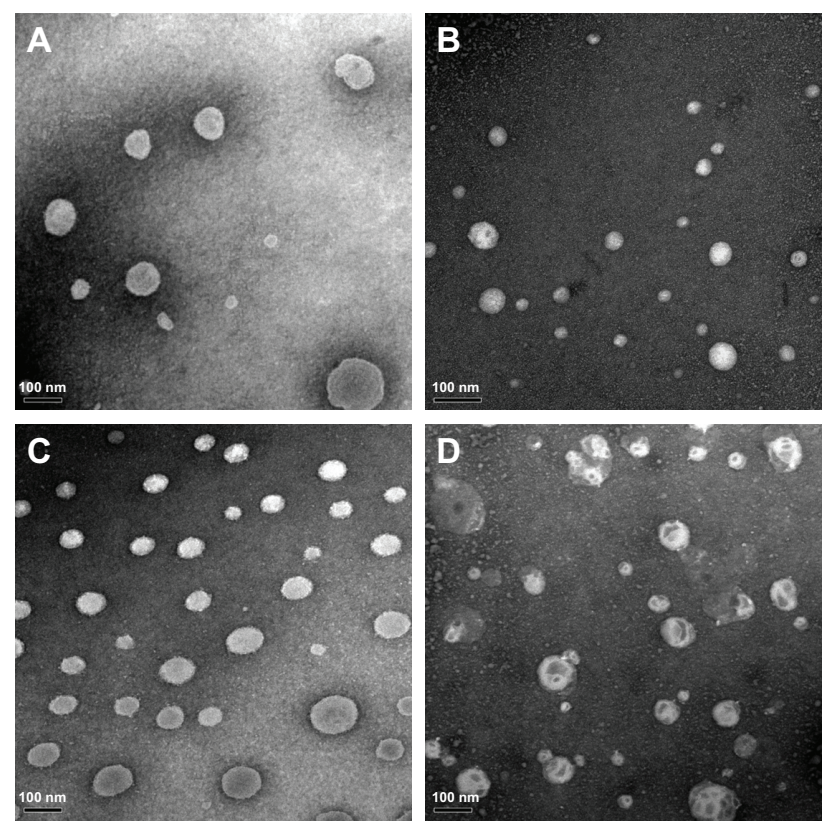

Figure 2 Morphology of tacrolimus-loaded liposomes containing sodium taurocholate (A), sodium deoxycholate (B), or sodium glycocholate (C), and conventional liposomes (D) by transmission electron microscopy.

was observed between liposomes containing different concentrations of sodium taurocholate, sodium glycocholate, or cholesterol. However, when the lipid concentration was more than $6 \mathrm{mg} / \mathrm{mL}$, the liposomes containing sodium deoxycholate showed more toxicity in SDHCECs than the $2 \mathrm{mg} / \mathrm{mL}$ concentration $(P<0.01)$, indicating that the cytotoxicity of liposomes containing sodium deoxycholate was greater than that of liposomes containing the other bile salts, ie, sodium taurocholate and sodium glycocholate.

Bile salts are a class of surfactants that cause irritation and toxicity to the cell membrane. ${ }^{25}$ Although some reports have shown that phospholipids can attenuate the toxicity of bile salts, ${ }^{26,27}$ the toxicity of liposomes containing bile salts has not been evaluated in detail for ophthalmic drug delivery systems. Liposomes containing sodium deoxycholate showed greater toxicity in SDHCECs than the other liposomes. The toxicity and surface activity of the bile salts varied according to their structures. Sodium deoxycholate incorporated in liposomes was particularly toxic to corneal cells, indicating that this bile salt is not a suitable enhancer for ocular drug delivery.

\section{Uptake by cells}

Uptake of tacrolimus-loaded liposomes by the SDHCECs is shown in Figure 5. Uptake increased with increasing incubation time for the four types of liposome formulation. Over a period of 2-8 hours, the cellular uptake of liposomes containing sodium glycocholate and those containing cholesterol was not significantly different, but uptake of liposomes containing sodium deoxycholate was less than that of those containing cholesterol during the time period of the study. However, by 12 hours, uptake of liposomes containing cholesterol was significantly greater than that of

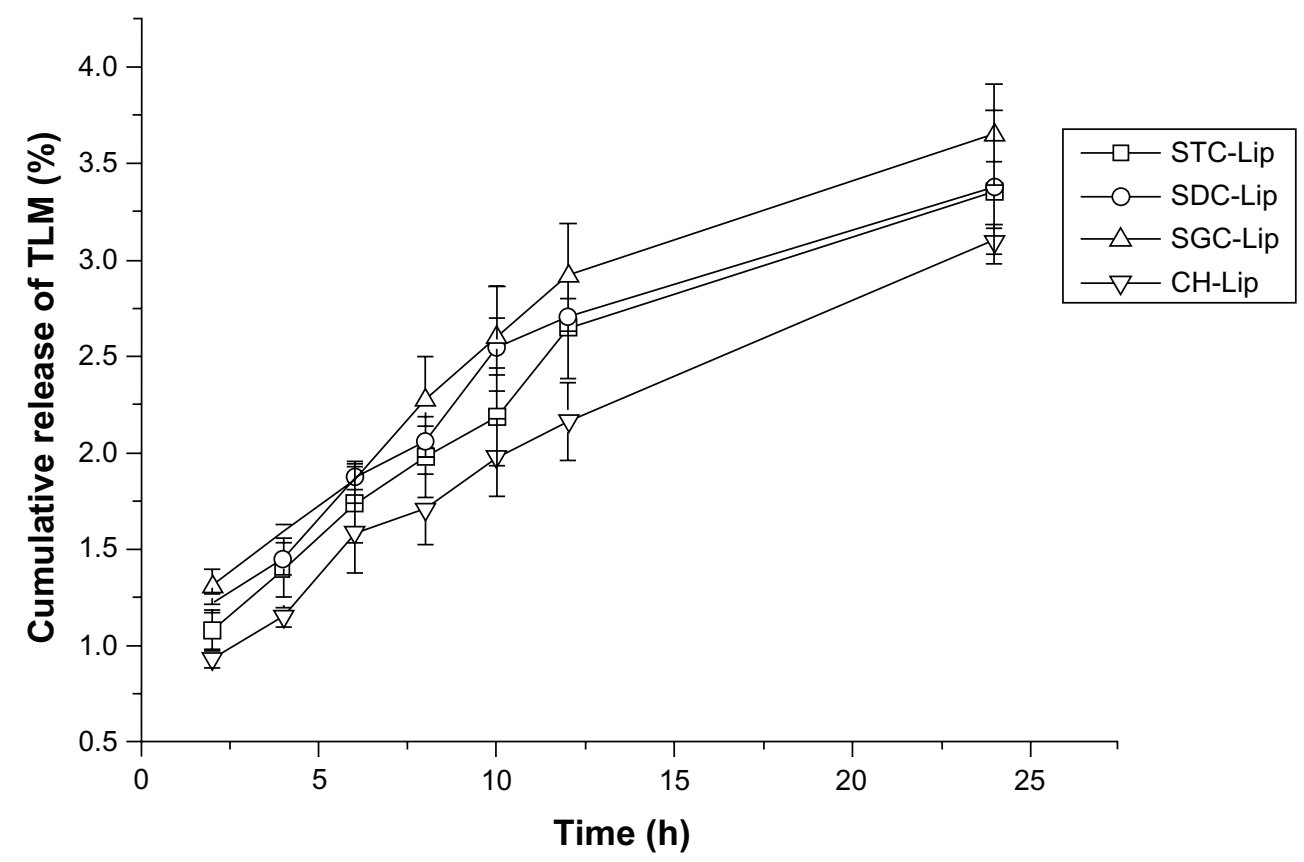

Figure 3 In vitro release profiles for tacrolimus (TLM) from liposomes (Lip) containing sodium taurocholate (STC), sodium deoxycholate (SDC), or sodium glycocholate (SGC), and conventional liposomes (cholesterol [CH]-Lip) in simulated tear fluid with $0.1 \%$ sodium dodecyl sulfate $(n=3)$. 


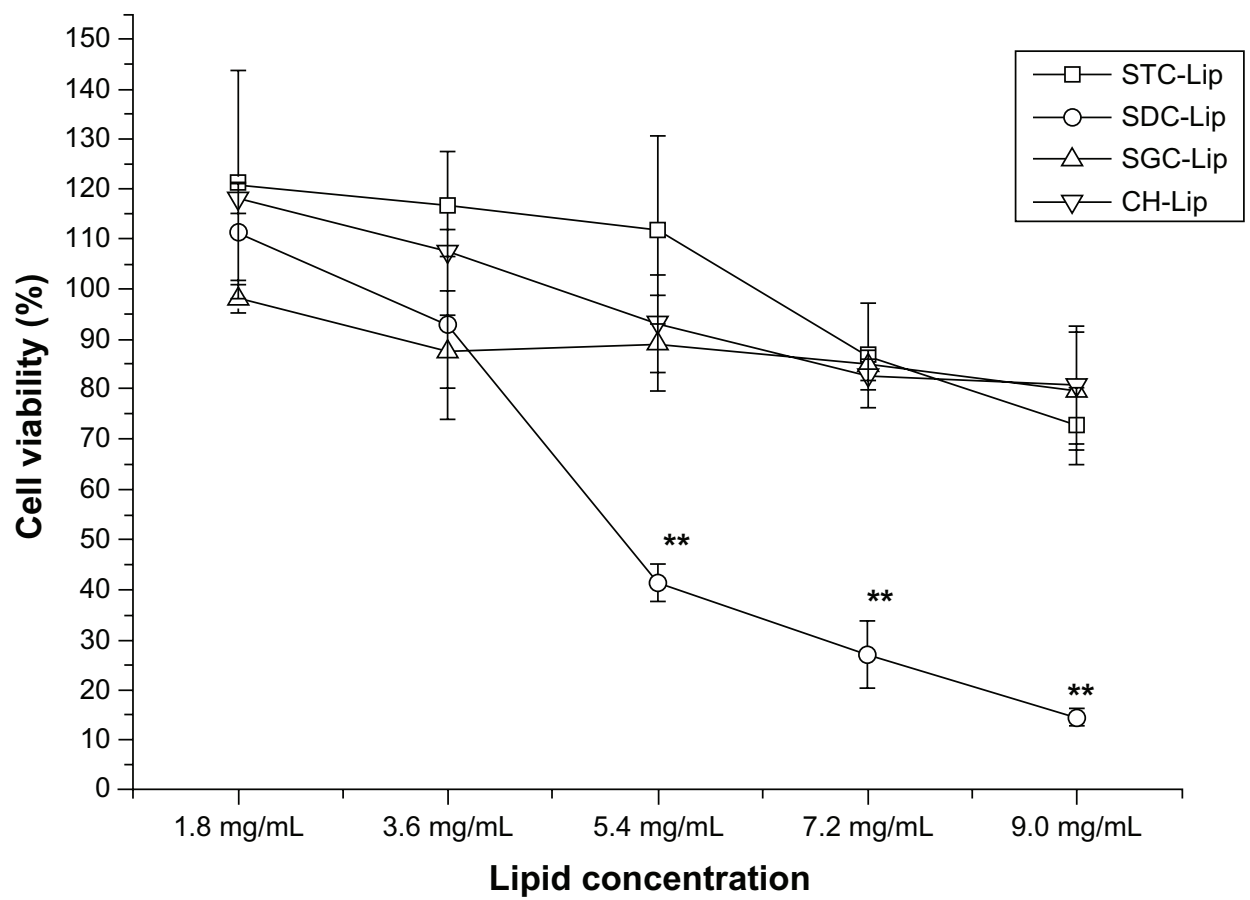

Figure 4 Cell viability measured by MTT after cells were exposed to different concentrations of liposomes (concentration calculated with soybean phosphatidylcholine) for 12 hours $(n=6)$.

Note: $* * P<0.01$ compared with conventional liposomes.

Abbreviations: CH-Lip, liposomes containing cholesterol; SGC-Lip, liposomes containing sodium glycocholate; SDC-Lip, liposomes containing sodium deoxycholate; STCLip, liposomes containing sodium taurocholate.

the liposomes containing bile salts, which may be a result of their negative zeta potentials.

Nanoparticles improve the penetration of drug molecules by various pathways, including increasing cellular uptake, improving membrane fluidity, and opening transient tight junctions. ${ }^{28}$ Therefore, we first studied the uptake of liposomes to understand the mechanism of improved uptake by cells. Liposomes are taken up by cells in a series of steps, including adsorption, fusion, and endocytosis. ${ }^{29}$ Cellular uptake was correlated with surface charge and

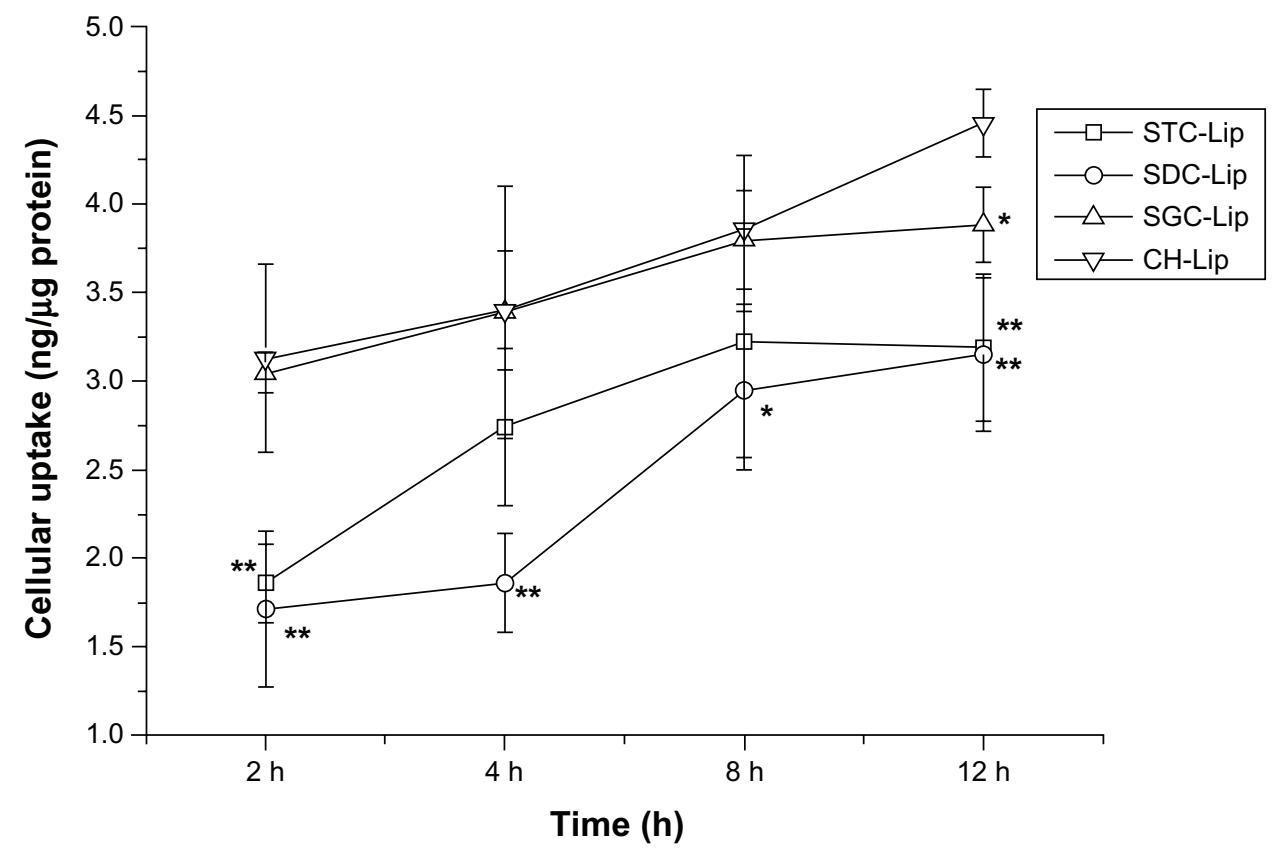

Figure 5 Cellular uptake of tacrolimus versus time after cells were incubated with tacrolimus-loaded liposomes (Lip) containing sodium taurocholate (STC), sodium deoxycholate (SDC), or sodium glycocholate (SGC), and conventional liposomes (cholesterol [CH]-Lip) $(n=3)$.

Notes: $* * P<0.01$ and $* P<0.05$ compared with conventional liposomes. 
increased with increasing positive charge. ${ }^{30}$ Therefore, the negatively charged nanoparticles adsorbed poorly onto the cell membrane, resulting in reduced SDHCEC uptake of liposomes containing bile salts compared with those containing cholesterol. However, cellular uptake of liposomes containing sodium glycocholate and those containing cholesterol was similar. This result agrees with our previous findings for insulin-loaded liposomes containing different bile salts. ${ }^{17}$

\section{In vitro transcorneal transport}

Figure 6 shows the in vitro corneal penetration results for liposomes containing sodium taurocholate, sodium deoxycholate, sodium glycocholate, or cholesterol. An almost linear relationship was observed between the amount of tacrolimus that had permeated through the cornea and time. The apparent permeability coefficients $\left(P_{\text {app }}\right)$ are shown in Table 2. When compared with liposomes containing cholesterol, those containing sodium taurocholate, sodium deoxycholate, or sodium glycocholate showed 4.5-fold, 3.7fold, and 3.7-fold increases in $P_{\text {app }}$, respectively, highlighting the enhanced penetration achieved by liposomes containing bile salts; however, there was no significant difference between liposomes containing the three different types of bile salts $(P>0.05)$. Although the liposomes containing bile salts have less affinity for the cell membrane, they are more flexible than those containing cholesterol, which favors their penetration through biomembranes. ${ }^{31,32}$ Moreover, bile salts can transiently open tight junctions to improve permeability of drug molecules or nanoparticles..$^{27,33}$

Careful handling of the isolated cornea and maintenance of its physiological activity throughout an experiment is very important for reproducibility of the results. Hydration level is a parameter frequently used to evaluate damage to the cornea. The normal cornea has a hydration level of approximately $76 \%-80 \%$, whereas an $83 \%-92 \%$ hydration level indicates damage to the epithelium and/or endothelium. ${ }^{34}$ In this study, the corneal hydration level ranged from $78.23 \% \pm 0.94 \%$ (for liposomes containing sodium taurocholate) to $81.59 \% \pm 2.31 \%$ (for liposomes containing sodium deoxycholate) and did not exceed $83 \%$, confirming the integrity of the corneas throughout the experiments.

\section{In vivo corneal uptake and transport}

Figure 7 shows images of corneal sections captured by confocal scanning laser microscopy after in vivo administration of the different types of liposome formulations when loaded with DiI perchlorate. DiI perchlorate is a fluorescent probe for the cell membrane, has very low aqueous solubility, and is commonly used to label liposomal membranes. ${ }^{35}$ No fluorescence was observed in the control corneal sections. Corneas from eyes treated with liposomes containing cholesterol emitted a more intense fluorescence signal in the epithelium than the other groups at 10 minutes after instillation. At 10 minutes, corneas from eyes treated

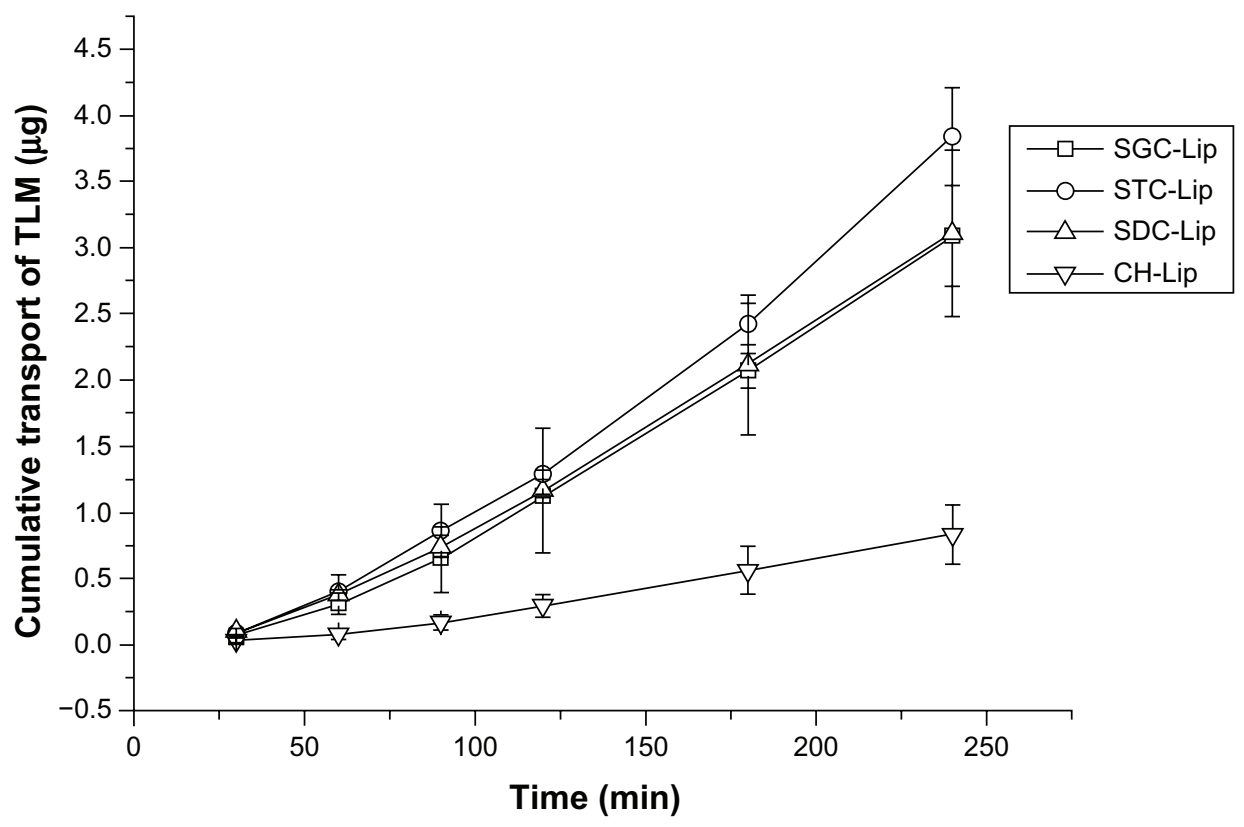

Figure 6 Cumulative transport of tacrolimus (TLM) across ex vivo rabbit corneas after administration of liposomes (Lip) containing sodium taurocholate (STC), sodium deoxycholate $(\mathrm{SDC})$, or sodium glycocholate $(\mathrm{SGC})$, and conventional liposomes (cholesterol $[\mathrm{CH}]$-Lip) at different time points $(\mathrm{n}=3)$. 
Table 2 In vitro transcorneal apparent permeability coefficients $\left(\mathrm{P}_{\text {app }}\right)$ of tacrolimus-loaded conventional liposomes and liposomes containing sodium taurocholate, sodium deoxycholate, or sodium glycocholate $(n=3)$

\begin{tabular}{lllll}
\hline Liposome formulation & STC-Lip & SDC-Lip & SGC-Lip & CH-Lip \\
\hline $\mathrm{P}_{\text {app }} \times 10^{8}(\mathrm{~cm} / \mathrm{sec})$ & $36.24 \pm 3.5 \mathrm{I}^{*}$ & $29.50 \pm 5.78^{*}$ & $29.73 \pm 4.03^{*}$ & $8.00 \pm 2.05$ \\
Fold increase & 4.5 & 3.7 & 3.7 & 1.0 \\
\hline
\end{tabular}

Note: $* P<0.01$ compared with $\mathrm{CH}$-Lip.

Abbreviations: $\mathrm{CH}$-Lip, liposomes containing cholesterol; SGC-Lip, liposomes containing sodium glycocholate; SDC-Lip, liposomes containing sodium deoxycholate; STC-Lip, liposomes containing sodium taurocholate.
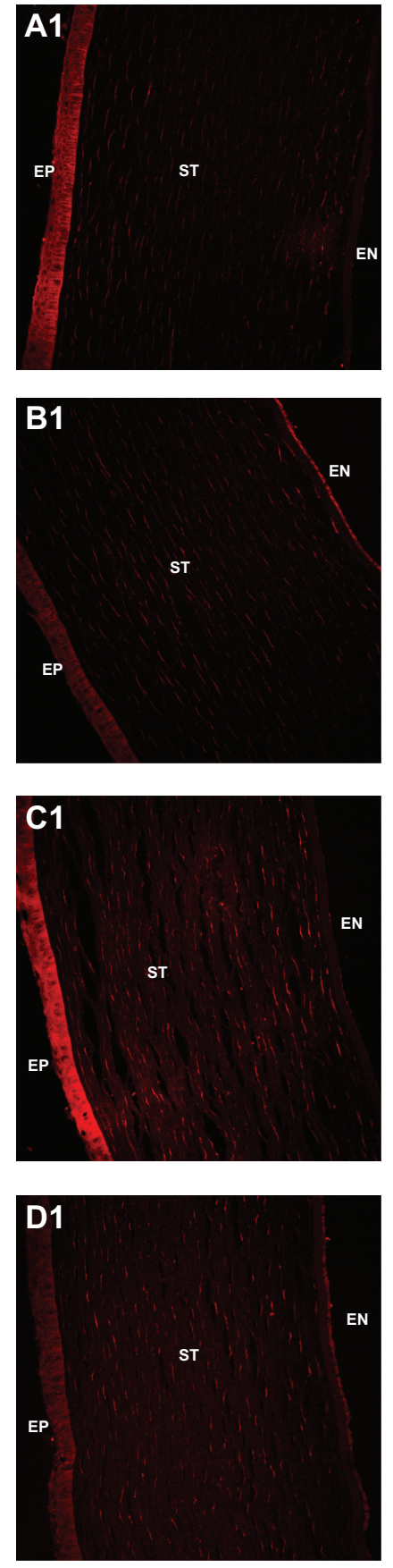
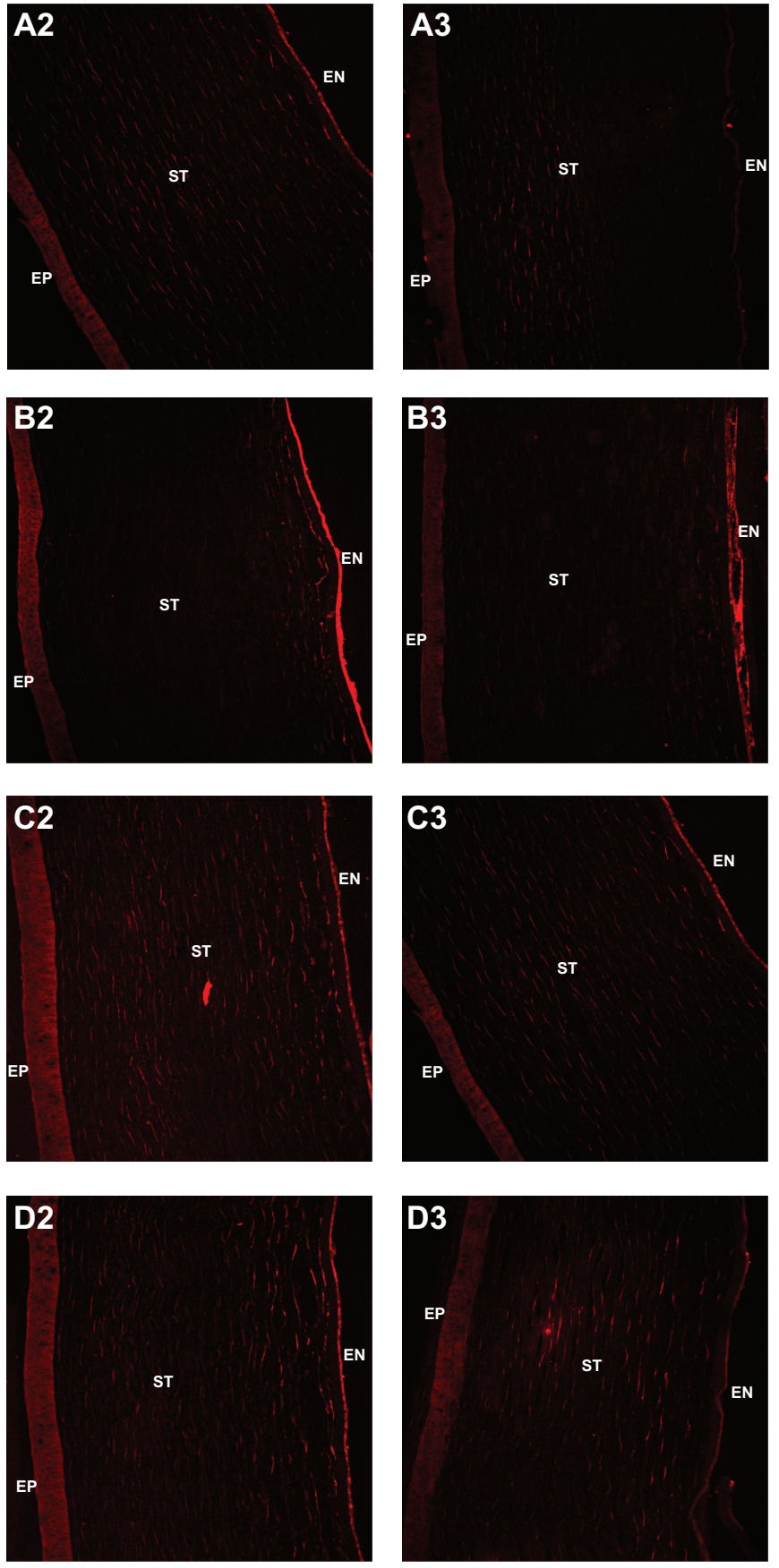

Figure 7 Confocal scanning laser microscopy of rabbit corneal sections at 10, 30, and 60 minutes after administration of conventional Dil perchlorate-loaded liposomes (AI-A3) and liposomes containing sodium deoxycholate (B I-B3), sodium glycocholate (CI-C3), or sodium taurocholate (DI-D3); I, 2, and 3 of each group are shown at 10,30 , and 60 minutes, respectively.

Abbreviations: EP, corneal epithelium; ST, corneal stroma; EN, corneal endothelium. 
with liposomes containing sodium deoxycholate or sodium taurocholate showed clear fluorescence signals in the endothelium, indicating rapid penetration of the vehicle. However, more intense fluorescence signals were observed in the corneal stroma from eyes treated with liposomes containing sodium glycocholate. After treatment with the liposomes for 30 minutes, fluorescence was observed in all of the corneal endothelial specimens, with the following order of intensity: liposomes containing sodium deoxycholate $>$ sodium taurocholate $>$ cholesterol $\approx$ sodium glycocholate. The stroma accumulated more of the liposomes containing sodium glycocholate. Moreover, 60 minutes after administration, very weak fluorescence signals were observed in corneas from eyes treated with the liposomes containing cholesterol. However, corneas treated with liposomes containing bile salts showed successive fluorescence signals from the epithelium to the endothelium, indicating that liposomes containing bile salts have stronger transcorneal penetration ability than those containing cholesterol.

Results from the in vivo corneal uptake experiments were consistent with our results for cell uptake and ex vivo corneal transport. DiI perchlorate is a commonly used fluorescence probe, which is hydrophobic and has a high affinity for the phospholipid membrane. ${ }^{36}$ Liposomes containing cholesterol could adsorb onto the corneal epithelium after instillation. Nevertheless, liposomes containing bile salts arrived at the endothelium through the stroma very rapidly, which can be attributed to their better transmembrane permeability. More liposomes containing taurocholate or sodium glycocholate distributed in the stroma and endothelium at 30 and 60 minutes after instillation. In vivo corneal uptake and transport results also demonstrate the improved ability of liposomes containing bile salts to permeate across the cornea.

\section{Corneal tolerance in vivo}

Figure 8 shows the corneal structures of untreated eyes (negative control) and eyes treated with liposomes or with $0.1 \%$ sodium dodecyl sulfate solution (positive control). Rabbit eyes treated with liposomes containing sodium taurocholate, sodium glycocholate, or cholesterol, as well as the negative control eyes showed no signs of discomfort during the six-hour assay. No differences in corneal structure were observed between eyes treated with liposomes containing sodium taurocholate, sodium glycocholate, or cholesterol and the negative control. However, when the corneal epithelium was exposed to $0.1 \%$ sodium dodecyl sulfate, the superficial epithelial cells were damaged and some gaps on the surface

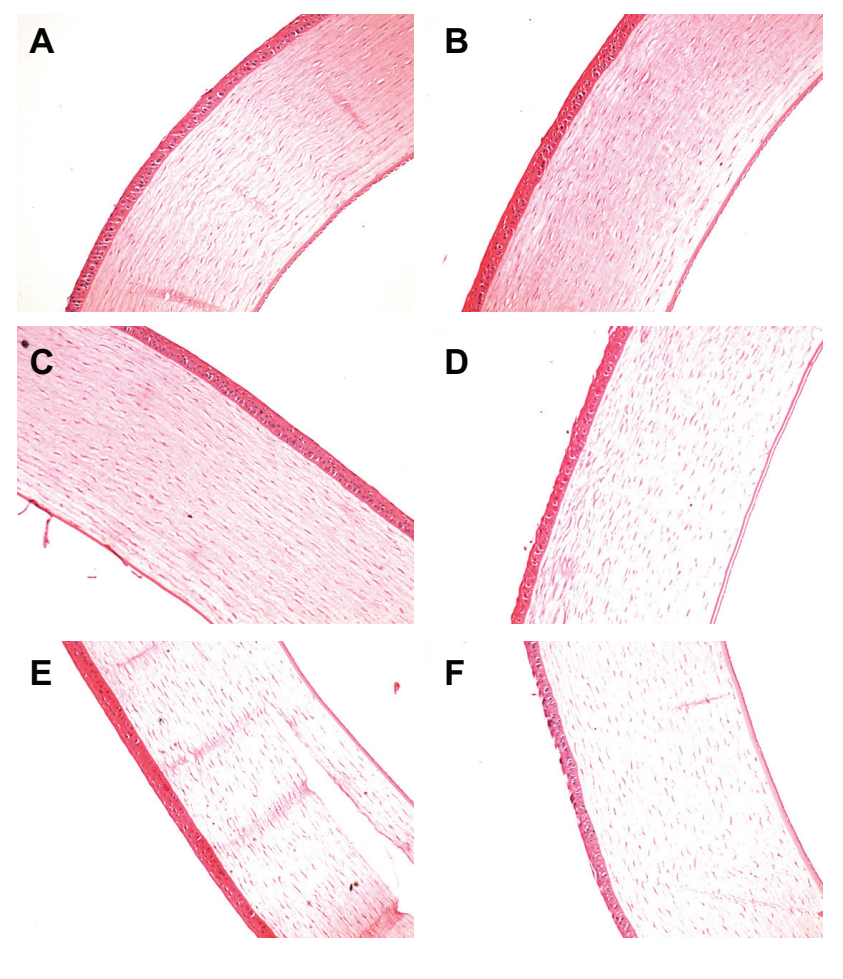

Figure 8 Histological cross-sections of excised rabbit cornea showing epithelium, stroma, and endothelium stained with hematoxylin and eosin after no treatment (A), administration of conventional liposomes (B) or liposomes containing sodium taurocholate $(\mathbf{C})$, sodium deoxycholate $(\mathbf{D})$, sodium glycocholate $(\mathbf{E})$, and $0.1 \%$ sodium dodecyl sulfate (F, positive control).

of the cornea were observed, indicating that sodium dodecyl sulfate is harmful to the rabbit cornea. Moreover, eyes treated with liposomes containing sodium deoxycholate showed gaps on the surface of the cornea, similar to those seen in the positive control group.

Although our cytotoxicity results indicate that liposomes containing sodium deoxycholate are more cytotoxic than the other liposomes, the actual situation in tissues may be different. Therefore, we studied corneal tolerance further after in vivo administration of the liposomes. Sodium dodecyl sulfate was chosen as a positive control because it can destroy most of the biomembrane due to its strong surface activity. ${ }^{9}$ We observed that liposomes containing sodium deoxycholate, but not the other liposomes, showed slight toxicity to the rabbit cornea, further indicating that sodium deoxycholate is not suitable for use in ocular drug delivery systems.

\section{Conclusion}

Liposomes containing cholesterol or bile salts, including sodium taurocholate, sodium deoxycholate and sodium glycocholate, were prepared using a thin-membrane dispersion method and showed similar particle size and entrapment efficiency. Release of tacrolimus from these 
liposomes was less than 5\% within 24 hours. Ex vivo corneal transport and in vivo corneal uptake experiments indicated that liposomes containing bile salts have better transmembrane permeation ability than those containing cholesterol and can improve the transport of tacrolimus across the cornea by 3-4-fold. Cytotoxicity and in vivo corneal tolerance studies indicated that liposomes containing sodium taurocholate or sodium glycocholate were well tolerated, whereas those containing sodium deoxycholate were toxic to both SDHCECs and rabbit cornea. Sodium taurocholate and sodium glycocholate incorporated into liposomes may be potential carriers for the ocular delivery of insoluble drugs such as tacrolimus.

\section{Acknowledgments}

This study was supported by the National Key Basic Research Program of China (2009CB930300). WW would like to thank the Shanghai Commission of Education (10SG05) and Ministry of Education (NCET-11-0114) for funding of personnel. We would like to thank Zhichong Wang from the Key Laboratory of Ophthalmology, Zhongshan Ophthalmic Center, for providing the SDHCECs, and Bo Tan for help with the high-performance liquid chromatography tandem mass spectrometry assay of tacrolimus.

\section{Disclosure}

The authors report no conflicts of interest in this work.

\section{References}

1. Inoue $\mathrm{K}$, Amano S, Oshika T, Tsuru T. Risk factors for corneal graft failure and rejection in penetrating keratoplasty. Acta Ophthalmol Scand. 2001;79:251-255.

2. Bourges JL, Lallemand F, Agla E, et al. Evaluation of a topical cyclosporine A prodrug on corneal graft rejection in rats. Mol Vis. 2006;12:1461-1466.

3. Hikita N, Lopez JS, Chan CC, et al. Use of topical FK506 in a corneal graft rejection model in Lewis rats. Invest Ophthalmol Vis Sci. 1997;38: 901-909.

4. Hewitt CW, Black KS. Comparative studies of FK506 with cyclosporine. Transplantation. 1988;46:482-483.

5. Birnbaum F, Reis A, Reinhard T. Long term use of topical tacrolimus (FK506) in high-risk penetrating keratoplasty. Cornea. 2009;28: 715-716.

6. Gao S, Sun J, Fu D, Zhao H, Lan M, Gao F. Preparation, characterization and pharmacokinetic studies of tacrolimus-dimethyl-beta-cyclodextrin inclusion complex-loaded albumin nanoparticles. Int J Pharm. 2012;427:410-416.

7. Berdoulay A, English RV, Nadelstein B. Effect of topical $0.02 \%$ tacrolimus aqueous suspension on tear production in dogs with keratoconjunctivitis sicca. Vet Ophthalmol. 2005;8:225-232.

8. Pleyer U, Lutz S, Jusko WJ, et al. Ocular absorption of topically applied FK506 from liposomal and oil formulations in the rabbit eye. Invest Ophthalmol Vis Sci. 1993;34:2737-2742.
9. Gan L, Han S, Shen J, et al. Self-assembled liquid crystalline nanoparticles as a novel ophthalmic delivery system for dexamethasone: improving preocular retention and ocular bioavailability. Int J Pharm. 2010;396:179-187.

10. Shen J, Sun M, Ping Q, Ying Z, Liu W. Incorporation of liquid lipid in lipid nanoparticles for ocular drug delivery enhancement. Nanotechnology. 2010;21:025101.

11. Chen Y, Lu Y, Zhong Y, Wang Q, Wu W, Gao S. Ocular delivery of cyclosporine A based on glyceryl monooleate/poloxamer 407 liquid crystalline nanoparticles: preparation, characterization, in vitro corneal penetration and ocular irritation. J Drug Target. 2012;20:856-863.

12. Hagigit T, Abdulrazik M, Valamanesh F, Behar-Cohen F, Benita S. Ocular antisense oligonucleotide delivery by cationic nanoemulsion for improved treatment of ocular neovascularization: an in-vivo study in rats and mice. $J$ Control Release. 2012;160:225-231.

13. Li N, Zhuang C, Wang M, Sun X, Nie S, Pan W. Liposome coated with low molecular weight chitosan and its potential use in ocular drug delivery. Int J Pharm. 2009;379:131-138.

14. Niu M, Lu Y, Hovgaard L, Wu W. Liposomes containing glycocholate as potential oral insulin delivery systems: preparation, in vitro characterization, and improved protection against enzymatic degradation. Int J Nanomedicine. 2011;6:1155-1166.

15. Guan P, LuY, Qi J, et al. Enhanced oral bioavailability of cyclosporine A by liposomes containing a bile salt. Int J Nanomedicine. 2011;6: 965-974.

16. Li L, Zhang Y, Han S, et al. Penetration enhancement of lidocaine hydrochloride by a novel chitosan coated elastic liposome for transdermal drug delivery. J Biomed Nanotechnol. 2011;7:704-713.

17. Niu M, Lu Y, Hovgaard L, et al. Hypoglycemic activity and oral bioavailability of insulin-loaded liposomes containing bile salts in rats: the effect of cholate type, particle size and administered dose. Eur $J$ Pharm Biopharm. 2012;81:265-272.

18. Paulsson M, Edsman K. Controlled drug release from gels using surfactant aggregates. II. Vesicles formed from mixtures of amphiphilic drugs and oppositely charged surfactants. Pharm Res. 2001;18:1586-1592.

19. Liu J, Song G, Wang Z, et al. Establishment of a corneal epithelial cell line spontaneously derived from human limbal cells. Exp Eye Res. 2007;84:599-609

20. Suhonen P, Jarvinen T, Peura P, Urtti A. Permeability of pilocarpic acid diesters across albino rabbit cornea in vitro. Int J Pharm. 1991;74: 221-228.

21. Baydoun L, Furrer P, Gurny R, Muller-Goymann CC. New surfaceactive polymers for ophthalmic formulations: evaluation of ocular tolerance. Eur J Pharm Biopharm. 2004;58:169-175.

22. Tammam S, Mathur S, Afifi N. Preparation and biopharmaceutical evaluation of tacrolimus loaded biodegradable nanoparticles for liver targeting. J Biomed Nanotechnol. 2012;8:439-449.

23. Seger C, Tentschert K, Stoggl W, Griesmacher A, Ramsay SL. A rapid HPLC-MS/MS method for the simultaneous quantification of cyclosporine A, tacrolimus, sirolimus and everolimus in human blood samples. Nat Protoc. 2009;4:526-534.

24. Hu S, Niu M, Hu F, et al. Integrity and stability of oral liposomes containing bile salts studied in simulated and ex vivo gastrointestinal media. Int J Pharm. 2013;441:693-700.

25. Heuman DM, Bajaj RS, Lin Q. Adsorption of mixtures of bile salt taurine conjugates to lecithin-cholesterol membranes: implications for bile salt toxicity and cytoprotection. J Lipid Res. 1996;37:562-573.

26. Dial EJ, Rooijakkers SH, Darling RL, Romero JJ, Lichtenberger LM. Role of phosphatidylcholine saturation in preventing bile salt toxicity to gastrointestinal epithelia and membranes. J Gastroenterol Hepatol. 2008;23:430-436.

27. Tan Y, Qi J, Lu Y, Hu F, Yin Z, Wu W. Lecithin in mixed micelles attenuates the cytotoxicity of bile salts in Caco-2 cells. Toxicol In Vitro. 2013;27:714-720.

28. Shahbazi MA, Santos HA. Improving oral absorption via drug-loaded nanocarriers: absorption mechanisms, intestinal models and rational fabrication. Curr Drug Metab. 2013;14:28-56. 
29. Khalil IA, Kogure K, Futaki S, Harashima H. Octaarginine-modified liposomes: enhanced cellular uptake and controlled intracellular trafficking. Int J Pharm. 2008;354:39-48.

30. Li Y, Wang J, Gao Y, Zhu J, Wientjes MG, Au JL. Relationships between liposome properties, cell membrane binding, intracellular processing, and intracellular bioavailability. AAPS J. 2011;13:585-597.

31. Gupta PN, Mishra V, Rawat A, et al. Non-invasive vaccine delivery in transfersomes, niosomes and liposomes: a comparative study. Int J Pharm. 2005;293:73-82.

32. Chen Y, Lu Y, Chen J, et al. Enhanced bioavailability of the poorly water-soluble drug fenofibrate by using liposomes containing a bile salt. Int J Pharm. 2009;376:153-160.

33. Chen X, Oshima T, Tomita T, et al. Acidic bile salts modulate the squamous epithelial barrier function by modulating tight junction proteins. Am J Physiol Gastrointest Liver Physiol. 2011;301:G203-G209.
34. Saettone MF, Chetoni P, Cerbai R, Mazzanti G, Braghiroli L. Evaluation of ocular permeation enhancer: In vitro effects on corneal transport of four [beta]-blockers, and in vitro/in vivo toxic activity. Int J Pharm. 1996;142:103-113.

35. Thongborisute J, Takeuchi H, Yamamoto H, Kawashima Y. Visualization of the penetrative and mucoadhesive properties of chitosan and chitosan-coated liposomes through the rat intestine. J Liposome Res. 2006;16:127-141.

36. Petersen S, Fahr A, Bunjes H. Flow cytometry as a new approach to investigate drug transfer between lipid particles. Mol Pharm. 2010;7: $350-363$.
International Journal of Nanomedicine

\section{Publish your work in this journal}

The International Journal of Nanomedicine is an international, peerreviewed journal focusing on the application of nanotechnology in diagnostics, therapeutics, and drug delivery systems throughout the biomedical field. This journal is indexed on PubMed Central, MedLine, CAS, SciSearch $®$, Current Contents ${ } /$ Clinical Medicine,

\section{Dovepress}

Journal Citation Reports/Science Edition, EMBase, Scopus and the Elsevier Bibliographic databases. The manuscript management system is completely online and includes a very quick and fair peer-review system, which is all easy to use. Visit http://www.dovepress.com/ testimonials.php to read real quotes from published authors.

Submit your manuscript here: http://www.dovepress.com/international-journal-of-nanomedicine-journal 